\title{
Imagerie SAR pour l'étude des processus côtiers
}

\author{
Hafedh Hajii, Sylvain Gouillon, Denis Bonicel \\ Ingénieurs MétéoMer
}

\begin{abstract}
Résumé
Ce papier a pour but de montrer la contribution du radar à ouverture synthétique (appelé SAR) à l'étude des processus côtiers. Il présente un exemple d'image SAR en zone littorale. La méthode exposée comprend une approche combinant l'imagerie SAR, les modèles numériques d'états de mer et de simulations de marées internes pour la détermination de paramètres dimensionnants.
\end{abstract}

\begin{abstract}
This paper intends to show the contribution of the Synthetic Aperture Radar (SAR) for studying the coastal processes. It shows an example of SAR image of internal waves and coastal transformation of swell waves and it contains a description of the MétéoMer operational models and the way to validate them.
\end{abstract}

\section{Introduction}

La caractérisation océano-météorologique de site, qu'il soit offshore ou côtier, consiste en la fourniture, aux ingénieries concernées, de paramètres dimensionnants de l'environnement marin, selon les trois axes du calcul de structure : statique, fatigue et dynamique (Charriez et al., 1995 et Hajji and Sproson, 1997). Les méthodes utilisées à MétéoMer reposent sur l'utilisation combinée des données sateilitaires, de modèles numériques de calcul d'états de mer au large et à la côte et d'outils pour l'analyse et l'extrapolation des données de vagues, vent et de courant.

Afin d'améliorer la phase de transfert à la côte (mer peu profonde) des états de mer calculés au large (mer profonde), MétéoMer développe des méthodologies d'utilisation des images SAR (Radar à Ouverture Synthétique) du satellite ERS. Récemment, plüsieurs schémas d'inversion ont été développés afin d'extraire des produits SAR mode vague au iarge (6kmx6km), les paramètres utiles d'états de mer (Bitner-Gregersen et al., 1996). En ce qui concerne le traitement des images SAR $(100 \mathrm{~km} \times 100 \mathrm{~km})$ en zones côtières, on doit prendre en compte des paramètres supplémentaires tels que la bathymétrie et les courants de surface.

D'autre part, pour des sites particuliers, des phénomènes de marées internes ont des effets non négligeables sur les opérations en mer. Ces ondes internes sont générées au niveau des talus continentaux, induites par la présence d'un courant barotrope et d'une thermocline océanique. La détection de ce type de courant à l'aide de l'observation satellitaire a fait l'objet de plusieurs publications scientifiques. Parmi celles-ci, l'étude d'Alpers (1985) constitue la base théorique de l'imagerie SAR des ondes internes.

Afin de caractériser le courant associé aux marées intemes, nous montrons un exemple d'utilisation d'image SAR en association avec un modèle numérique de simulation de ces courants. 


\section{Caractérisation des états de mer côtiers}

\subsection{Etat de l'art et objectifs}

\subsubsection{Imagettes et images SAR}

Les données SAR en mode vague sont des spectres d'imagettes $(6 \mathrm{Km} 6 \mathrm{Km})$. Afin d'extraire les caractéristiques de l'état de mer (Hs, Tz, $\theta$ pic) correspondant, il est nécessaire d'effectuer l'inversion (Bitner-Gregersen et al., 1996). Cette méthode est utilisée en raison du caractère nonlinéaire des relations entre la surface de la mer et le spectre d'image correspondant. Plus particulièrement, les spectres d'images SAR ont les caractéristiques suivantes: informations comprises entre 7 et 25 secondes tous les $15^{\circ}$, ambiguilté de $180^{\circ}$ sur la direction; manque ou perte d'informations pour les périodes en dessous de los le long de la trace du satellite; différences éventuelles sur la localisation des pics d'énergie.

L'extraction des spectres de vagues en zone côtière est souvent plus complexe du fait de l'influence sur les états de mer d'autres paramètres tels que la bathymétrie (réfraction/shoaling), le frottement sur le fond et les interactions avec les courants. De même, les longueurs de stationnarité en zones côtières sont plus courtes qu'en mers ouveries et de ce fait, l'état de mer contenu dans üne surface de $6 \mathrm{~km} \times 6 \mathrm{~km}$ n'est pas homogène.

Les objectifs des développements en cours sont de faire évoluer le schéma d'inversion des spectres SAR (Bitner-Gregersen and al., 1996) aux zones côtières. Pour les états de mer non stationnaires sur des surfaces de $6 \mathrm{kmx} 6 \mathrm{~km}$, il s'agira de développer une méthode complémentaire permettant l'extraction de certains paramètres (Tpic, $\theta$ pic,...).

Ces développements concerneront les images SAR puisqu'il est possible, à partir d'une surface de $100 \mathrm{Km} \times 100 \mathrm{Km}$, d'étudier à la fois les paramètres d'états úe mer au large et à la côte.

\subsubsection{Les modèles numériques MétéoMer}

A MétéoMer, l'analyse et la prévision des états de mer est une activité importante. Deux modèles d'états de mer sont opérationnels. Le premier est utilisé pour le large (ISFS-DW) et le second pour les zones côtières (ISFS-SW).

Les champs de vent utilisés proviennent des modèles météorologiques tels que ARPEGE ou ECMWF. Afin d'améliorer ces champs de vent, MétéoMer utilise toutes les informations disponibles et notamment :

- Satellites géostationnaires : METEOSAT, NOAA, GOES, GMS,

- Modèles d'analyse Allemand, Anglais et Russe,

- Satellites défilants : MétéoMer est connecté à l'Agence Spatiale Européenne via ISDN et reçoit toutes les données LBR (Low Bit Rate) incluant celles de l'altimètre (Hs et force du vent), du SAR en mode vague (traités par la suite à l'aide d'un schéma d'inversion) et du diffusiomètre (champs de vent).

Les modèles ISFS sont utilisés, en temps réel pour l'assistance aux travaux en mer, et en temps différé pour les études climatologiques. 


\section{a) Modèle d'états de mer au large : ISFS-DW (Integrated Swell Forecasting System/Deep Water) :}

ISFS est un modèle spectral de vague. Les spectres directionnels sont représentés par un nombre discret de bandes spectrales se propageant sur un nombre donné de directions. Ceci conduit à une représentation détaillée de l'état de mer pour un lieu donné en terme de spectre bi-dimensionnel (fréquence - direction).

Les principaux termes de ce modèle ISFS sont donnés par :

$\partial / \partial \mathrm{t} E(f, \theta, \mathbf{x}, \mathrm{t})+\mathbf{C}_{\mathbf{g}} \cdot \nabla \mathrm{E}=\mathrm{S}(\mathrm{f}, \theta, \mathbf{x}, \mathrm{t})$

$E$ est la densité d'énergie du champ de vague décrit comme étant fonction de la fréquence $f$, de la direction $\theta$, de la position $\mathbf{x}$ et du temps $t, \mathbf{C}_{\mathrm{g}}$ est la vitesse de groupe du champ de vague en eau profonde. Le terme $\mathrm{C}_{\mathrm{g}} . \nabla \mathrm{E}$ représente l'advection théorique et le terme $\mathrm{S}$ représente les fonctions sources incluant la génération et la dissipation.

L'équation (1) est habituellement résolue par différences finies pour une série de pas de temps, sur une grille couvrant le bassin océanique étudié. Dans ISFS, cette équation est calculée le long de lignes d'orthodromies convergeant vers le point de calcul.

\section{b) Modèle côtier : ISFS-SW Antegrated Swell Forecasting System/Shallow Water) :}

Le modèle côtier MétéoMer permet le transfert effectif des états de mer calculés au large. Ce modèle peut être aussi utilisé pour générer des vagues en zones côtières.

L'équation (1) est écrite sous la forme :

$\partial / \partial \mathrm{t} \mathrm{E}_{\mathrm{ij}}+\partial / \partial \mathrm{s}\left(\mathbf{C}_{\mathrm{g}} \cdot \mathrm{E}_{\mathrm{ij}}\right)=\mathrm{I}+\mathrm{W}+\mathrm{D}$

où $\partial / \partial \mathrm{t}$ est le taux de variation le long de la direction de propagation de la composante $E(\mathrm{i}, \mathrm{j})$. Le terme $\partial / \partial s\left(\mathbf{C}_{\mathrm{g}} E_{\mathrm{ij}}\right)$ représente l'advection d'énergie sur le point de calcul. Cela représente les effets du gradient spatial de la densité spectrale, les effets de modification de la vitesse de groupe avec la modification de la profondeur (shoaling) et le changement de direction (réfraction).

Les termes sources comprennent la génération de vagues par le vent locai (I), les interactions nonlinéaires vague-vague (W) et la dissipation par frottement sur le fond et/ou le déferlement (D).

Le modèle ISFS-SW utilise la même approche que le modèle du large, résolvant la conservation de l'énergie des vagues (l'action en présence de courant) sur les raies de rétropropagation.

Les champs de vent et les simulations des modèles numériques de vagues sont validés grâce à l'utilisation des données sateilitaires .

\subsection{Applications}

L'image SAR ERS-1 de la Figure 1 a été prise en Angola le 23 Juillet 1994. Les deux isobathes superposées sur l'image correspondent aux sondes 200 et 40 mètres. Cette image couvre donc une zone profonde $(500 \mathrm{~m})$ ainsi qu'une zone de petite profondeur. La vitesse du vent est inférieure à $5 \mathrm{~m} / \mathrm{s}$ et la zone noire correspond à des vent très calmes. De plus, trois lignes parallèles aux isobathes, correspondant à des ondes internes, sont visibles sur cette image. 


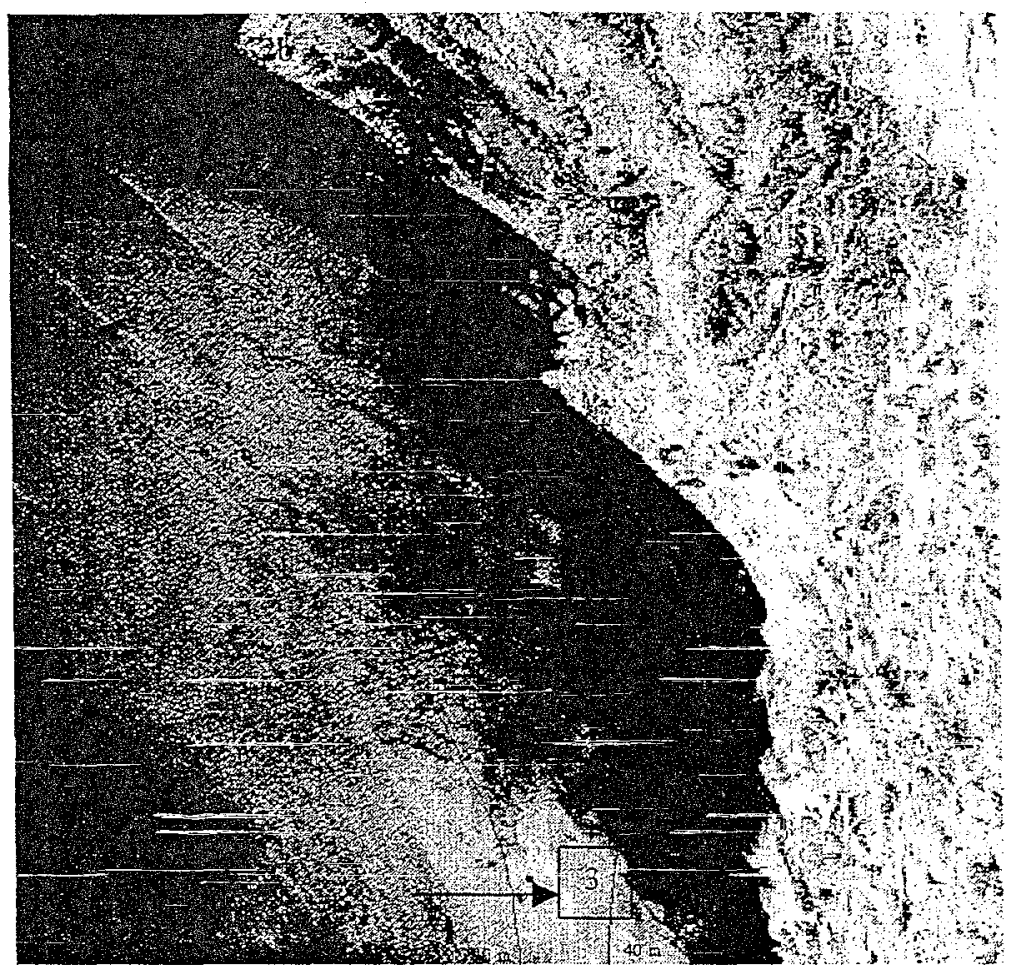

Figure 1 : Image SAR en Angola le 23 Juillet 1994. Les zones noires correspondent à des vents très calmes. Des ondes internes sont visibles sur les parties inférieures et supérieures de l'image. Les figures (2a) et (2b) correspondent aux imagettes respectivement au large et à la côte. Les deux lignes continues correspondent aux isobathes 200 et 40 mètres. Les flèches montrent des paquets de solitons.

Afin de montrer la potentialité des images SAR pour l'étude des transferts côtiers, deux imagettes sont extraites (figures $2 \mathrm{a}$ et $2 \mathrm{~b}$ ). La figure $2 \mathrm{a}$ illustre les conditions d'états de mer au large quand la houle est bien «rangée». A la côte, les vagues deviennent plus courtes et tournent vers l'Est (figure 2b).

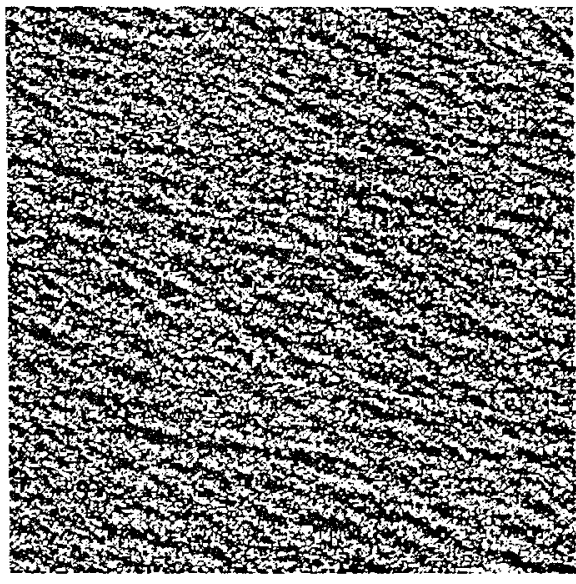

Figure 2.a : exemple d'imagette montrant le système de houle

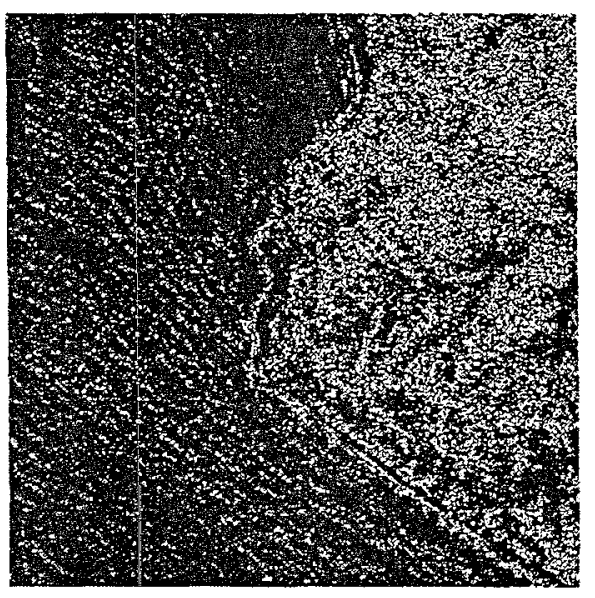

Figure 2.b : exemple d'imagette montrant la réfraction/diffraction du fait de la bathymétrie et la présence du cap 


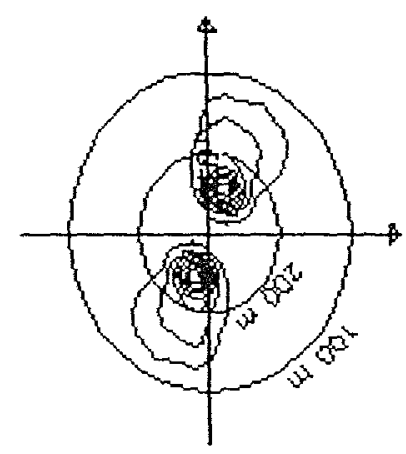

Figure 2.c: Spectre d'image calculé à partir de l'imagette située en mer profonde. Le pic correspond à une longueur d'onde de $400 \mathrm{~m}$, les vagues (houle) se propagent vers le Nord-Est.

L'inversion du spectre au large donne une longueur d'onde pic de 370 mètres avec une direction de $215^{\circ}$, le $\mathrm{Hs}$ est de $1.84 \mathrm{~m}$. En zone côtière, ces caractéristiques sont altérées et plus particulièrement celles relatives au vecteur d'onde. En effet, la longueur d'onde pic est de 200 mètres et de direction $235^{\circ}$.

\section{Caractérisation de la marée interne}

\subsection{Etat de l'art et objectifs}

Les ondes internes de marée sont générées par le passage de la marée barotrope sur des discontinuités topographiques telles que le talus continental lorsque l'océan est stratifié Mazé, 1987). La maree interne ainsi générée se propage principalement dans les zones à forts gradients de densité comme la thermocline saisonnière. Les courants de surface associés aux ondes internes induisent des champs rugueux en surface (champs générés par les interactions entre les ondes courtes de surface engendrées par le vent et les courants induits en surface par les solitons) permettant ainsi leur détection par les radars (Alpers, 1985).

Les courants associés aux ondes internes peuvent avoir des conséquences sur les opérations en mer. Par exemple, la perturbation de la thermocline engendre des modifications de propagation des ondes acoustiques. De même, les variations brusques de courant sur toute la tranche d'eau induisent des effets dommageables sur les structures offshore (Osborne and Burch, 1980).

Les objectifs de MétéoMer sont de valider un modèle numérique de génération d'ondes internes permettant l'analyse et la prévision de ces phénomènes à l'aide des images SAR. 


\subsection{Modèle de génération d'ondes internes}

Pour simuler la génération d'ondes internes due à la présence d'un courant barotrope, un modèle à deux dimensions a été choisi (Hibiya, 1988). Sous les approximations de Boussinesq, les équations de mouvements et de continuité dans un fluide incompressible peuvent être écrites sous la forme suivante :

$\mathrm{D} u / \mathrm{D} t=-1 / \rho_{0} \partial \mathrm{p} / \partial \mathrm{x}+\mathrm{A}_{\mathrm{h}} \partial^{2} u / \partial \mathrm{x}^{2}+\mathrm{A}_{\mathrm{v}} \partial^{2} u / \partial z^{2}$

$\mathrm{D} w / \mathrm{D} t=-1 / \rho_{0} \partial \mathrm{p} / \partial z-\left(\rho / \rho_{0}\right) g+\mathrm{A}_{\mathrm{h}} \partial^{2} w / \partial x^{2}+\mathrm{A}_{\mathrm{v}} \partial^{2} w / \partial z^{2}$

$\mathrm{D} \rho / \mathrm{D} t=-w \partial \rho_{0} / \partial z$

$\partial u / \partial x+\partial w / \partial z=0$

où $t$ est le temps; $u$ et $w$ sont respectivement les vitesses du fluide dans les directions $\mathrm{x}$ et $\mathrm{z}$; $\mathrm{D} / \mathrm{D} t=\partial / \partial t+\mathrm{u}(\partial u / \partial x)+w(\partial / \partial z)$ est la dérivation totale en fonction du temps; $\rho_{0}$ est la densité de l'eau dans un état d'équilibre; $\rho_{0}$ est la moyenne de $\rho_{0}$ : $\rho$ est la perturbation de la densité de l'eau ; $p$ la pression; $g$ l'accéiération due à la gravité, $A_{h}$ et $A_{v}$ sont respectivement les coefficients horizontaux et verticaux de viscosité tourbillonnaires.

En introduisant la fonction de courant $\Psi$ définie par :

$\mathrm{u}=\partial \psi / \partial \mathrm{z}$

$w=-\partial \psi / \hat{\partial x}$

(1)-(4) conduisent aux équations suivantes:

$\mathrm{D} \xi / \mathrm{D} t=\mathrm{g} / \rho_{0} \partial \rho / \partial x+A_{\mathrm{h}} \partial^{2} \xi / \partial x^{2}+A_{\mathrm{v}} \partial^{2} \xi / \partial z^{2}$

$\mathrm{D} \rho / \mathrm{D} t=\partial \psi / \partial x \partial \rho_{0} / \partial z$

$\nabla^{2} \psi=\zeta$

où $\nabla^{2}$ est l'opérateur de Laplace, $\nabla^{2}=\partial / \partial x^{2}+\partial^{2} / \partial z^{2}$

La variable d'itération est $\zeta$ et les conditions aux limites sont données par :

$\psi=0 \quad$ pour $\quad \mathrm{z}=-\mathrm{H}(x)$

$\psi=\mathrm{Q}(t) \quad$ pour $\quad \mathrm{z}=0$

où $\mathrm{Q}(t)$ est le flux de volume de marée barotrope, $z=\mathrm{H}(x)$ est l'équation de la profondeur.

Les dernières équations sont résolues à l'aide d'un schéma aux différences finies avec variables centrées et un schéma «saute-mouton» avec un incrément $\Delta t$ sur le temps.

Ce modèle a été choisi essentiellement en raison de l'aspect non-linéaire des solitons et de par sa simplicité d'application. 


\subsection{Applications}

Si les ondes internes détectées par le SAR (voir flèches sur la figure 1) ont été générées par la marée, marée semi-diume dans cette région, l'estimation de la distance entre les deux paquets situés au nord de l'image donnerait une vitesse de propagation de l'ordre de $0.3 \mathrm{~m} / \mathrm{s}$.

Afin d'analyser visuellement les ondes internes détectées par le SAR en mode image, une extraction d'imagette, en haute résolution, a été effectuée sur l'une d'entre elles. La figure 3 présente l'onde interne (paquet de solitons) située dans le bas de l'image montrée en figure 1. Cette zone correspond probablement à une zone de génération d'onde interne de marée du fait de la topographie. La réalisation d'une coupe le long du paquet de solitons permet d'estimer la longueur d'onde, celle-ci est de 200 mètres environ (figure 4).

Ces résultats d'analyse de l'image serviront de référence pour la validation du modèle de génération (voir section 3.2) d'ondes internes.

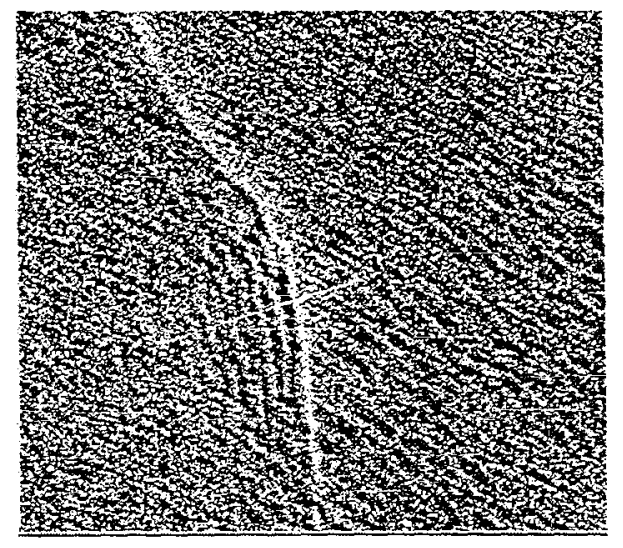

Figure 3: imagette $6 \mathrm{~km} \times 6 \mathrm{~km}$ montrant un paquet de solitons

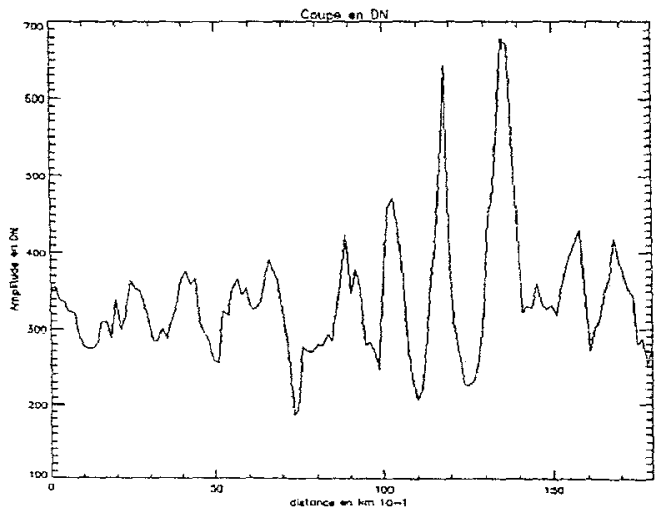

Figure 4: profil le long du paquet de solitons

\section{Conclusion}

Les images SAR foumissent une bonne opportunité d'améliorer le transfert à la côte et les modèles de générations d'ondes internes. Les exemples montrés ici illustrent le potentiel des images SAR pour analyser les phénomènes de réfraction/diffraction et d'anomalies de courant.

Actuellement, les développements effectués ont permis d'extraire les principaux paramètres associés aux images SAR et aux processus côtiers. Ainsi, nous nous rapprochons des objectifs d'utilisation opérationnelle de ces produits satellitaires à fort potentiel.

\section{Remerciements}

Ce travail à été effectué dans le cadre d'un projet pilote (A-O-L.F201) de l'Agence Spatiale Européenne. MétéoMer remercie l'ESA pour la fourniture de l'image SAR traitée ici. 


\section{Références}

Alpers $W$ :

Theory of radar imaging of internal waves.

Nature, $314: 245-247,1985$.

Bitner-Gregersen E, D. Bonicel., H. Hajji., M. Olagnon and G. Parmentier :

Word-wide characteristics of $\mathrm{Hs}$ and $\mathrm{Tz}$ for long-term load responses of ships and offshore structures.

Proc. Int. Offshore and Polar Engineering Conf., ISOPE, 1996.

Brandt $P$. and $W$. Alpers :

Validation of numerical model describing the generation and propagation of internal waves in the strait of Gibraltar by using ERS-1 Synthetic Aperture Radar data.

Proc. Of the Second ERS-1 Symposium. ESA SP-361. 1993.

Charriez $P ., H$. Hajji and $V$. Thowenin :

Combined use of satellite data for a reliability approach of design wave estimation.

Proc. Int. Offshore Mechanics and Arctic Engineering, OMAE, 1995.

Hajji H. and R.A.Sproson:

Satellite measurements and their application to the Pechora sea.

Proc. Int. Offshore and Polar Engineering Conf., ISOPE 1997.

Hibiya T:

The generation of internal wave by tidal flow over Stellwagen Eank.

J.Geophys.Res., 93, NO.C1,533-542, 1988.

Mazé, R. :

Generation and propagation of non-linear internal waves induced by tide over a continental slope. Cont. Shelf Res., 7(9):1079-1104, 1987.

Osborne, A., R., and T.L. Burch:

Internal solitons in the Andaman Sea.

Sciences, vol. $208,451-460,1980$.

Pichon A. and R. Mazé :

Internal Tides over a shelf break : analytical model and observations.

Amer. Met. Soc, 20, 657-671, 1990. 